\title{
La medicalización de la infancia como estrategia de gobierno
}

\author{
Mauricio Bedoya-Hernández' \\ Pablo Andrés de Francisco-León ${ }^{2}$ \\ Lina Julietd Mesa-Osorio ${ }^{3}$
}

\section{Resumen}

El presente artículo informa de la investigación que tuvo como objetivo conocer las estrategias psicocientíficas asociadas a la producción de subjetividad en el neoliberalismo. El método usado fue el histórico-crítico fundado en los estudios foucaultianos. Resultados: ( I) la medicalización de la vida ha sido una estrategia de gobierno; (2) la medicalización de la infancia - también una estrategia de gobierno - ha pasado por tres momentos, cada uno de los cuales produce una forma específica de subjetividad; (3) se hallan tres razones para medicalizar la infancia y (4) las psicociencias y las biociencias son los discursos que han dirigido esta medicalización. Se concluye que el neoliberalismo ha usado esta estrategia de medicalización elaborada por las ciencias psi para ampliar la mercantilización de la vida y configurar la subjetividad autogestionada del emprendedor.

Palabras clave: Medicalización. Infancia. Subjetividad. Neoliberalismo.

\section{Introducción}

Con su reflexión sobre la mayoría de edad en Was ist Aufklarung?, Kant (2009) se atrevió a lo que hasta ese momento la filosofía no había hecho: un diagnóstico de su presente. Foucault resalta cómo Kant fue el primero en hacer una ontología de nosotros mismos $(1999,2009)$, lo cual será la base de lo que, en la actualidad, se denomina historia del presente. Problematizar lo

I Licenciado en Educación, Psicólogo, Magíster en Psicología, Doctor en Ciencias sociales. Profesor de la Facultad de ciencias sociales, Universidad de Antioquia (Medellín, Colombia). E-mail: mauricio.bedoya@udea.edu.co

2 Estudiante de filosofía, Universidad de Antioquia (Medellín, Colombia). E-mail: pablo.defrancisco@udea.edu.co

3 Estudiante de psicología, Universidad de Antioquia (Medellín, Colombia). E-mail: julietd.mesa@udea.edu.co

\section{$(\mathrm{cc}) \mathbf{B r}$}

Direito autoral e licença de uso: Este artigo está licenciado sob uma Licença Creative Commons. Com essa licença você pode compartilhar, adaptar, para qualquer fim, desde que atribua a autoria da obra, forneça um link para a licença, e indicar se foram feitas alterações. 
que somos, preguntarnos cómo hemos llegado a serlo y de qué manera somos gobernados a partir de ello es el cometido de esta historia crítica del presente. Por gobierno entendemos, con Foucault (2001), la conducción de la conducta de los otros, lo que deriva en la estructuración de su posible campo de acción.

Para efectos del tema en el presente artículo, partimos de una triple caracterización de nuestro presente. En primera instancia, el mundo contemporáneo está regido por una forma de gubernamentalidad neoliberal (FOUCAULT, 2007; LAVAL; DARDOT, 2013, 2017; BROWN, 2017). El neoliberalismo es una racionalidad de gobierno que busca la construcción de una subjetividad determinada, la del neosujeto (LAVAL; DARDOT, 2013), empresario de sí mismo, individuo que asume su vida como una empresa mediante un incesante trabajo de autogestión con el fin de incrementar ilimitadamente su capital humano. Para el neoliberalismo, a diferencia del liberalismo clásico, todas las esferas de la vida individual y social se reducen a la variable económica y al mercado.

En segunda instancia, el neoliberalismo hace una gestión medicalizada de la vida del neosujeto. Aunque la medicalización de la vida no es una estrategia gubernamental nueva - existe desde hace más de doscientos años -, en el presente asume rasgos distintivos importantes, como conducir a que todos los aspectos de la vida del individuo, sano o enfermo, sean interpretados bajo la lógica de los ejes normalidad-anormalidad y saludenfermedad. El emprendedor, que vive en continua competencia con los demás, también empresarios de sí, se ve amenazado por esos otros; por lo tanto, se siente en riesgo continuo y aprende a vivir en riesgo, pero, como dice Sennett (2000), eso corroe su carácter y lo enferma. La medicalización opera aquí en cuatro sentidos: el individuo se siente insuficiente al compararse con sus competidores (siempre habrá alguien mejor), se lee como anormal, se enferma (al exigirse más de lo que sus límites le permiten dar) y se engancha en el mercado del mejoramiento continuo mediante el consumo de productos como la autoayuda, la psicofarmacología, la consejería, las asesorías de imagen, los cursos de empleabilidad, entre otros, con el fin de ser el mejor empresario de sí.

En tercera instancia, esta medicalización de la vida fomenta la creciente medicalización de la infancia dentro del neoliberalismo. Hay 
una serie de razones para probarlo, como mostraremos más adelante. Una de ellas es que el neoliberalismo ve en la infancia un gran nicho de mercado por explotar. En el presente texto nos interesa, en particular, el proceso de medicalización de la vida y la infancia que las psicociencias - denominación dada por Rose (1996) a los saberes científicos psi como el psicoanálisis, la psiquiatría, la psicología - han elaborado, y que el neoliberalismo ha usufructuado para lograr sus fines.

Tanto para Stolf y Caponi (2013) como para Sy (2018), la medicalización de la vida es un proceso por el cual son definidos en términos médicos los comportamientos, las acciones y los rasgos individuales. Esta forma de definición de lo humano exige una lectura desde la terminología médica y prepara escenarios para la intervención de los aspectos patológicos. Nosotros proponemos que, para pensar el problema de la medicalización de la vida y de la infancia como una estrategia gubernamental contemporánea, son necesarias tres consideraciones: por una parte, la medicalización excede a la medicina como arte que busca curar la enfermedad somática. Por otra parte, gracias al concurso de las psicociencias, esta noción adopta el razonamiento médico de base para llevarlo a aspectos de la vida que no son somáticos. Finalmente, la medicalización adquiere, gracias a las psicociencias, un papel central en la conducción de la totalidad de la existencia de los individuos. Como puede colegirse, no negamos la existencia de la psicopatología infantil ni del sufrimiento que acarrea para los niños y sus familias; tampoco negamos la existencia de comportamientos desviados al igual que lo hacen Stolf y Caponi (2013). Pero creemos que la gestión positiva de la vida y, dentro de ella, de la enfermedad psicológica, se debe al neoliberalismo, para cuyo fin confisca la estrategia gubernamental de la medicalización infantil.

\section{Metodología}

Como lo explicamos antes, Michel Foucault reconoce a Kant como el primer filósofo que se preguntó por su presente y que, al mismo tiempo, vio su actualidad como acontecimiento. Kant inaugura una línea de trabajo que Foucault denominó ontología del presente u ontología de nosotros mismos (1994, 2009), la cual propende por convertir el presente 
en fuente de problematización y crítica. Basado en esto, el presente estudio adopta la metodología histórico-crítica, propia de la historia del presente de inspiración foucaultiana, la cual, en decir de Dreyfus y Rabinow (2001), debido a su carácter genealógico, se interroga por lo que somos hoy y por los sistemas de normas y verdades que nos gobiernan.

Nuestra metodología partió de cuestionarnos por lo que somos hoy. Somos sujetos medicalizados por la psicociencias y, por consiguiente, gobernados por el neoliberalismo. La siguiente pregunta genealógica a la que respondimos fue “¿cómo llegamos a ser lo que somos hoy?" Esto nos guio hacia una indagación histórico-crítica de la medicalización de la vida emprendida por las ciencias psi desde el siglo XIX hasta nuestros días. La tercera pregunta que incluye nuestra ruta analítica fue "¿de qué manera emergió la medicalización de la infancia?” Aquí hallamos que se produjo como una derivación de la medicalización de la vida cuando la gubernamentalidad liberal, y posteriormente la neoliberal, localizó a la infancia como foco de gobierno. La especificidad del neoliberalismo es que, además de lo anterior, hizo de la infancia un nicho de mercado. Finalmente, nuestra cuarta pregunta genealógica aludió a qué forma de subjetivación produce la medicalización de la vida en general y de la infancia, en particular, en el contexto amplio del gobierno de los individuos y las sociedades.

Dado que este estudio, por su carácter genealógico, se interesa por la relación entre regímenes discursivos de las psicociencias, sistemas normativos y formas de subjetividad - todo ello en el contexto del gobierno neoliberal -, el concepto de subjetivación es central. Pero es necesario aclarar que Foucault pensó de dos maneras la subjetivación. Hasta el Nacimiento de la biopolitica, la subjetivación se refiere a la manera como el neoliberalismo produce un tipo de subjetividad, la del sujeto emprendedor (URABAYEN; CASERO, 2018; CASTRO-GÓMEZ, 2016, 2010; FOUCAULT, 2007). Así que el motor de la subjetivación es el gobierno (neoliberal). Pero, en los cursos posteriores, Foucault desarrolla la pregunta acerca de si es posible hacerse un tipo de sujeto que se gobierna a sí mismo como forma de resistirse al gobierno neoliberal (CASTRO-GÓMEZ, 2016; DELEUZE, 2015; FOUCAULT, 1998). Como se aprecia, nuestro estudio se focaliza en la subjetividad producida por el neoliberalismo. Queda por estudiar 
la cuestión de si es posible hacerle frente, y resistirse, a la medicalización contemporánea explotada por el neoliberalismo para gobernarnos a nosotros mismos.

\section{La medicalización de la vida como estrategia de gobierno}

\section{I De la ausencia de cuerpo a la ciudadanía biológica}

La psiquiatría inicial fue incapaz de localizar en el cuerpo la enfermedad mental a pesar de sus esfuerzos (FOUCAULT, 2005; CAPONI, 2009a; CASTEL, 2009). Sus preguntas por el tejido, el órgano o el sistema perturbado en la locura influyeron poderosamente en la fisiología y la psicología tempranas. La investigación respecto de la localización cerebral de las facultades mentales se mantiene desde Gall, pasando por Broca (CANGUILHEM, 1998), hasta nuestro presente.

Sin un cuerpo anatomopatológico para la enfermedad mental, los psiquiatras hallaron en la familia del loco su cuerpo ampliado, y en la herencia, un elemento explicativo clave (CAPONI, 2009a; FOUCAULT, 2005). Con la reconstrucción de la historia de vida del sujeto a partir de sus antecedentes (BEDOYA, 2018), se consolidaron el interrogatorio, la medicación y la hipnosis como instrumentos básicos de indagación.

La historia íntima se convirtió en materia explicativa de la enfermedad mental. Según lo elaborado por el psicoanálisis desde sus inicios, ella define y conduce la vida individual. Eventualmente, se tornó en foco de gobierno. En este contexto es que emerge el mercado psi con sus servicios terapéuticos diversos. Hasta nuestros días, la tarea del sujeto psi consiste en hacer del pasado un campo de búsqueda para lograr el mayor bienestar posible mediante ejercicios de gobierno (ROSE, 1996; BEDOYA, 2018).

La psiquiatría del siglo XIX hizo converger la familia, la historia personal y la psicopatología, mientras que el psicoanálisis aportó densidad teórica. También fue determinante la psiquiatría ampliada, y en especial, la teoría de la degeneración (CAPONI, 2009b). Consideramos que sus efectos fueron consolidar la psiquiatría en su labor de gobierno e incluir la 
vida individual y la intimidad en la mirada psiquiátrica. Así confluyeron la medicalización de la vida intima y el gobierno de los ciudadanos, es decir, la conducción de la conducta mediante el discurso psiquiátrico y psicológico (en el siglo XIX) y psicoanalítico y psicoterapéutico (desde el siglo XX).

Las psicociencias, unidas a esa lectura psiquiátrica-médica de sí mismo, se convirtieron en productoras de nuevos diagnósticos a partir de la patologización de la experiencia individual (BARCALA, 2015). Mediante la teoría de la degeneración moreliana, Kraepelin sentó las bases para la psiquiatría moderna y la medicalización de las vivencias existenciales y sociales (CAPONI, 2010a, 2012a, 2012b), de la subjetividad y lo social (BEDOYA, 2018). Con el discurso biopolítico del riesgo de contraer enfermedades mentales aparecieron las subjetividades conformadas para no enfermar. Así, la psiquiatría y la psicología quedan constituidas como tecnologías biopolíticas desde finales del siglo XIX.

Con Kraepelin se potenciaron los sistemas clasificatorios de los trastornos mentales basados en la anatomopatología cerebral, la herencia y la estadística (CAPONI, 2010b). El uso de la estadística para efectos comparativos y evaluativos le suministró a la psiquiatría el anhelado cuerpo de la enfermedad mental al relacionarla con aspectos biológicos y sociales acordes al imperativo biopolítico. Aquí se gestaron las estrategias técnicoconceptuales que permitirán a las psicociencias del siglo XX intervenir los desvíos comportamentales. La biologización de lo social realizada por la psiquiatría (CAPONI, 2010a, 2012a, 2012b) y las psicociencias tomó forma hace poco más de cien años. Rose (2012) destaca la importancia de la genética, las neurociencias y la biotecnología en la configuración de la subjetividad del presente y la emergencia de la denominada ciudadanía biológica (ROSE; NOVAS, 2004), la cual hizo de la vitalidad y del cuerpo somático el núcleo de subjetivación.

Sostenemos como hipótesis que una de las principales estrategias de gobierno de las psicociencias es la medicalización de la vida. Encontramos una primera instancia de medicalización con intencionalidad biopolítica en el arreglo familia-hijos, precisamente en el ámbito de la medicina social y la nosografía del siglo XVIII, cuando el saber médico se incrustó en la 
vida de las poblaciones (FOUCAULT, 1985, 1996). A mediados del siglo pasado surgió una suerte de somatocracia que ya había sido insinuada; el Estado hizo del cuerpo de los individuos un foco de cuidado y salud permanente. Esto tendrá profundos efectos posteriores: la medicalización, desde el siglo XX, abarca la vida en su totalidad hasta tornarse indefinida. Consecuentemente, "la medicina se impone a los individuos como un acto de autoridad" (CASTRO, 2011, p. 273).

\subsection{Estrategias para medicalizar la vida}

Como lo hemos expuesto antes (BEDOYA, 2018), las psicociencias, comprometidas con el gobierno de los individuos y las poblaciones, han participado en la medicalización de la vida mediante cuatro estrategias: (1) la psicologización de los individuos y de la vida social; (2) la elaboración de un lenguaje que le permite al sujeto nombrarse a sí mismo; (3) la patologización de la vida; (4) la psicofarmacologización.

\subsection{Psicologización de la subjetividad}

Con la psicologización del yo, Álvarez-Uría (2006) propone que la interioridad se instala como núcleo de la definición de sí mismo a lo largo del siglo XX. Más allá del individualismo moderno, en la última centuria ocurre una primera concordancia indefectible entre interioridad y subjetividad alrededor del yo psicológico. El psicoanálisis, al robustecer el sujeto psi decimonónico propio de la psiquiatría y la psicología con la idea de que el individuo es gobernado por un mundo interno insondable (BEDOYA, 2018), permitió una segunda concordancia entre subjetividad e historia personal. Mundo interno e historia personal permanecen como marcas de la subjetividad hasta nuestros días.

Coincidimos con Foucault (1996, p. 53) cuando plantea que, si bien el psicoanálisis es la primera antipsiquiatría, "[...] no se logra salir de la medicalización y todos los esfuerzos en este sentido se remiten a un saber médico" al ser un tratamiento psíquico aunado a la interpretación de la subjetividad desde lo normal y lo anormal (ILLOUZ, 2007, 2010). Por esto, discrepamos de Castel (1986) cuando anuncia el fin de la hegemonía del discurso psicoanalítico: la cultura sigue definiéndose desde las certezas que produce el mundo interno y el papel determinante de la infancia y el inconsciente. 
En este contexto puede entenderse que el culto al yo (ÁLVAREZ-URÍA, 2006) se haya fortalecido: del elogio a la individualidad, la autonomía y la libertad promulgado en el siglo XIX, se ha pasado a su uso por parte del gobierno neoliberal del presente. Proponemos que el neoliberalismo, enfocado en la capitalización de suvida, usa el cultoal yo para configurar sujetos emprendedores. Como consecuencia, el individuo se convierte en la única explicación de su propio padecimiento. $Y$ si añadimos el problema contemporáneo de la política (LAVAL; DARDOT, 2013; HAN, 2014; ÁLVAREZ-URÍA, 2006), ese yo neoliberal psicologizado termina por despolitizarse voluntariamente. El sujeto empresario rompe con la preocupación por lo común (LAVAL; DARDOT, 2015), la mutualidad y la solidaridad (BROWN, 2017) al hacer de los otros sus competidores o clientes.

El neosujeto, como designan Laval y Dardot (2013) al sujeto neoliberal, encuentra en su salud y habilidades psicológicas un capital humano imprescindible para su labor. Formarse es un imperativo del empresario de sí y una responsabilidad individual con sus respectivos costes. El neosujeto cae en una remodelación indefinida de su yo que lo conduce a engancharse en el mercado psi de la autoestima, el control emocional, el optimismo, la felicidad y otras habilidades. En consecuencia, el mercado del automejoramiento, conducido por el discurso psicocientífico, se fortalece; las variables psicológicas quedan definidas como ejes de interpretación de la propia subjetividad y de la empresarialidad de sí (BEDOYA, 2018). Los criterios de automejoramiento tendrían dos procedencias: el régimen discursivo de las psicociencias y las exigencias propias del mercado empresarial de sí mismo. Visto desde los ideales modernos de autonomía y libertad, esto no es más que la normalización que hace el individuo de los requerimientos del mercado (CASTEL, 1986).

Diversos sectores de las psicociencias han participado en la constitución de esta racionalidad de gobierno de los individuos del presente. Al psicologizar lo social, el sujeto se autorresponsabiliza sin mirar las causas sociales e institucionales de su padecer, se ausculta sin parar, convirtiéndose en experto lector de sus desviaciones e incompetencias. La vida social es leída en clave de psicología individual (DUEÑAS, 2019).

Aunque coincidimos con la idea de psicologización de la vida expuesta por Castro Orellana (2014), nuestra hipótesis es que tal procedimiento 
hace parte del proceso de medicalización de la subjetividad, que incluye la creación de un lenguaje especializado para hablar de sí mismo.

\subsubsection{Construcción de un lenguaje experto}

Las psicociencias construyen un sistema de verdad sobre el sujeto que dirige la actuación de los individuos. Este régimen veridiccional, clave en la estrategia de psicologización de la subjetividad, se concreta en un lenguaje para hablar de sí mismo y autorreconocerse. Creado a inicios del siglo XX, el lenguaje psi colonizó todas las esferas de la vida individual y social, y ha sido recreado incesantemente para gobernar la vida pública y privada.

Este lenguaje determina ejes para que el sujeto se comprenda, se nombre, se evalúe y se reconozca ante sí y ante los demás. Dicho proceso, denominado vectorización de sí (BEDOYA, 2018), lleva al individuo a juzgarse según su localización en el continuo entre normalidad y anormalidad psicológica. Para Rose (2008), las ciencias psi crearon un sentido común en Occidente.

Afirmamos que el lenguaje psi tiene cinco usos:

(1) Hace posible que el sujeto se reconozca y se nombre ante sí y ante los otros.

(2) Permite advertir nuestros malestares, pero, al mismo tiempo, produce dos deslizamientos: considerarlos como desviaciones e interpretarlos como patologías psíquicas originadas de traumas infantiles, exceso de estrés, baja autoestima, pensamientos inadecuados, etc. (BARCALA, 2015; ILLOUZ, 2010).

(3) Refiere a estrategias correctivas de la psicopatología: psicoterapia, autoayuda, coaching, entre otras. Esto muestra la vocación normalizadora que las ciencias psi mantienen desde su nacimiento (FOUCAULT, 2005; CASTEL, 1986).

(4) Delimita los focos de gobierno subjetivo; es decir, los aspectos de la vida que han de ser objeto de atención e intervención por parte de las sociedades (BARCALA, 2015) y los individuos mismos. Las ciencias psi construyen un régimen de verdades con el propósito de ofrecer claves para intervenir $y$, de ese modo, concretar su vocación correctiva. 
(5) Impulsa a que cada individuo emprenda acciones sobre esos focos de gobierno, reconduciendo su vida y, de paso, modificando la relación consigo mismo. A esto Foucault $(1998,1999)$ lo denomina ética. Así, el lenguaje psi es usado para configurar una experiencia ética.

Con su deslizamiento malestar-desviación-psicopatología, el lenguaje experto hace que el malestar subjetivo sea medicalizado y, bajo esta condición, reconduce a los sujetos hacia conductas tenidas por saludables

Clarificamos que, si bien ese lenguaje experto es creado por los psicocientíficos, su uso no es exclusivo de ellos, puesto que lo comparten con los administradores de la vida de sus dirigidos (padres de familia, docentes, gerentes, gobernantes y demás). Esto es lo que Rose (1996) llama la generosidad del discurso psi.

\subsubsection{Lectura de la vida como patología}

En Foucault (2005) y Caponi (2009a) leemos que el problema para las psicociencias decimonónicas fue la ausencia de cuerpo de la enfermedad mental y, en consecuencia, de un diagnóstico absoluto. Por esto, los psicocientíficos adoptaron criterios importados de múltiples disciplinas para precisar la existencia de la enfermedad mental, produciendo una ola imparable de sistemas de clasificación de los trastornos mentales hasta el más reciente Manual diagnóstico y estadístico de los trastornos mentales (DSM) publicado por la Asociación Americana de Psiquiatría.

La pregunta “¿qué es la enfermedad mental?”, difícil de responder para las psicociencias, derivó en la creación de la función (FOUCAULT, 2005) y el sujeto psicológico. Lo psicológico fue la tecnología conceptual inventada para dicha cuestión (BEDOYA, 2018). Análogamente, en las psicociencias se gestó desde el principio una confusión entre normas vitales y normas sociales. Como lo hemos señalado (BEDOYA, 2018), en este contexto emergió la quirúrgica de la subjetividad, una intervención correctiva de la vitalidad somática promovida desde el modelo médico (ROSE, 2001, 2012).

El éxito de las psicociencias contemporáneas es que se han presentado como autoridades indispensables de diagnóstico, clasificación y tratamiento. Separan al sujeto autorrealizado y feliz de aquel no-realizado y desviado (CABANAS; ILLOUZ, 2019) usando la emocionalidad como criterio de 
jerarquización de los sujetos (ILLOUZ, 2007). Gracias a la popularización del imperativo de la autorrealización por parte de Carl Rogers y Abraham Maslow (ILLOUZ, 2007; AMORÓS, 1996; MASLOW, 1989), terminaron asimilándose autorrealización y bienestar personal ilimitado con salud mental. No obstante, los creadores de esta noción no le dieron un contenido específico y delimitado (ILLOUZ, 2007, 2010). Cada individuo tiene que descubrir su potencial y su destino; de no hacerlo, será catalogado como enfermo. El temor a ser una persona frustrada acabó por estimular la patologización de la vida cotidiana. La exigencia de autorrealizarse se volvió un instrumento para normalizar. El neoliberalismo lo usa para configurar el neosujeto al convertir la autorrealización en desarrollo de competencias para el emprendimiento y la empresarialidad, y medicaliza la vida logrando que el neosujeto se perciba en riesgo de no realizarse debido al lastre traumático de su historia personal, que amenaza el bienestar total y la búsqueda de la felicidad. El neoliberalismo une experiencias incompatibles - vivir en riesgo y hacerse emprendedor - para crear un mercado de automejoramiento constante.

En la historia de Occidente se han considerado riesgosas experiencias humanas como el deseo, las pasiones, los sentimientos y las emociones (GARCÍA, 2006; FOUCAULT, 1998, 2002). Las formas de afrontarlas han

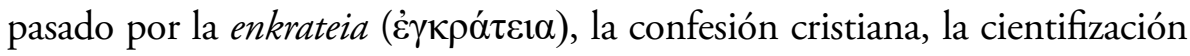
moderna de la vida y el mercado psi contemporáneo. Antagónicamente, el presente hace un llamado a exteriorizar las emociones y regularlas mediante la idea de que hacen parte del capital humano. El individuo medicalizado se hace consumidor del mercado de mejoramiento de sí y la salud mental queda convertida en mercancía (PAPALINI, 2013; ILLOUZ, 2007). Hoy se le demanda al sujeto ser exitoso para que con ello muestre su salud mental.

Pero el neosujeto no está condenado. Si bien el neoliberalismo hace que el individuo se viva como sujeto irrealizado y en continuo riesgo, lo conduce también a familiarizarse con su desviación psicológica, de modo que se inserte en el mercado del mejoramiento ilimitado gestionándose positivamente (BEDOYA, 2018).

\subsubsection{Psicofarmacologización del yo}

La estrategia de patologización producida por las psicociencias lleva a que aspectos cotidianos de la vida sean incluidos dentro del arreglo 
normalidad-patología cuando antes no lo eran (BEDOYA, 2018; BARCALA, 2015; CABRAL DE BARROS, 2010; CAPONI, 2010b; MARTÍNEZ, 2010). Al interpretar estratégicamente toda desviación como psicopatología (ROSE, 1996) se allana el camino para la creación sin fin de categorías nosológicas y para ejercer un control difuso, pero ilimitado, de los individuos y las poblaciones.

El lenguaje experto es un instrumento básico para identificar a los desviados y, al mismo tiempo, para gobernar la subjetividad. El individuo desviado es impelido a vigilarse continuamente. Las psicociencias pasaron del disciplinamientoliberalalautocontrolneoliberal(BEDOYA;CASTRILLÓN, 2018). Dada la generosidad de las psicociencias, hoy todos somos expertos psi, diagnosticadores de la subjetividad propia y ajena. Canguilhem (1998) habla de la "cuesta abajo" de la psicología - que consideramos extensible a todas las ciencias psi -, para referirse a la vigilancia que este saber despliega sobre la subjetividad humana. Las psicociencias se ilusionan con otorgarle cuerpo a la enfermedad mental mediante el conocimiento profundo de la estructura cerebral (ROSE, 2012; CANGUILHEM, 1980) con la meta de conocer la verdad sobre el sujeto y las claves para dirigir su existencia.

El aumento de valoraciones psicopatológicas y la ampliación de los manuales diagnósticos de trastornos mentales coincide con la proliferación de tratamientos psicofarmacológicos (FERNÁNDEZ, 2014; PAPALINI, 2013). En el presente se gobierna a los sujetos y a las poblaciones mediante una falacia medicalizadora. A cambio de ofrecer el psicofármaco como remedio a la enfermedad mental, suceden dos contrariedades: (1) el medicamento atenúa algunos síntomas produciendo otros inesperados sin curar la afección; (2) el sujeto queda enganchado a estas sustancias, temeroso e inseguro de enfermar más si las abandona. Con ello gana la industria psi y la industria farmacéutica (ROSE, 2001, 2012; CAPONI, 2009c, 2010a; FORCADES, 2006; PIGNARRE, 2005).

Al señalar que "[...] el antidepresivo tiene un efecto doble: permite definir la etiología (ISRS-déficit de serotonina) y permite optimizar y crear nuevos criterios diagnósticos" (CAPONI, 2009c, p. 336) nos admite colegir que los psicofármacos han influido poderosamente en la creación de criterios diagnósticos y de nuevas patologías presentadas al público como naturales. 
Debido al alarmante incremento en el uso de fármacos, Laval y Dardot (2013, p. 372) señalan que en la sociedad contemporánea presenciamos un "dopaje generalizado". Al sujeto se le demanda rendir ilimitadamente, ser exitoso y convertirse en un competente empresario de sí; aquí hallamos el caldo de cultivo para su padecimiento y para el recurso a los fármacos, vistos como un sostén necesario para vivir. Gotzsche (2014) expone que la medicalización de la cotidianidad de las personas está siendo usufructuada y promovida por la industria farmacéutica. Para este autor, la corrupción de dicha industria es evidente, aduciendo sobornos a psicocientíficos, gobernantes y medios de comunicación, además de oscuros procedimientos investigativos, manipulación de la información divulgada, promesas a potenciales consumidores de que el medicamento mejorará su vida.

La confianza en las promesas de la industria farmacéutica y los profesionales psi es la clave del éxito de la farmacologización de la subjetividad (BEDOYA, 2018). El diseño de la subjetividad ofrecido por el neoliberalismo en asocio con amplios sectores científicos y de la industria de los medicamentos, pero también deseado por el sujeto contemporáneo, es lo que denominamos quirúrgica del yo. Este diseño del yo prometido por los psicofármacos ataca aspectos considerados patológicos (ROSE, 2001, 2012) y aspectos que, si bien son vistos como sanos, podrían ser potenciados. Pareciera crecer la vulgata acerca de que estas sustancias mejoran el desempeño del individuo en todos los niveles al aumentar su rendimiento y optimizar su portafolio subjetivo, movilizando la población al consumo indiscriminado de fármacos. A esto lo denominamos ética de la promesa y se vincula con la economía politica de la esperanza (ROSE, 2012), que justifica el autocontrol y el restablecimiento del verdadero yo.

Hemos pasado del encierro manicomial al "encerramiento químico" (CAPONI, 2009a, p. 102). Comolo muestran Castel, Castely Lovell (1980), con la aparición de los psicofármacos se consolidó la crítica al manicomio y la consecuente desinstitucionalización de los enfermos mentales. La cura se focalizó en las "drug-maintenance clinics, es decir consultas de mantenimiento bajo medicación” (CASTEL, CASTEL; LOVELL, p. 91). En este sentido hemos de comprender que el "encerramiento químico" y el "dopaje generalizado" tienen una relación de reciprocidad con 
el "yo neuroquímico" y la "ciudadanía biológica” (ROSE, 2012). Lo que se juega aquí no es solo la eficacia de los psicofármacos, sino la manera como estos dirigen la vida del individuo y, sobre todo, la relación que el sujeto establece consigo mismo cuando considera usarlos. Como lo enuncia Rose (2012. p. 433): “[...] en la constitución molecular de esas drogas hay una ética incorporada: las drogas mismas plasman e incitan formas particulares de vida en las que 'el yo real' es tanto 'natural' como producido".

\section{Medicalización de la infancia: del niño traumatizado al niño autogestionado.}

Sy (2018) ve la medicalización de la infancia como una práctica que patologiza la conducta infantil y las diversas esferas de esta etapa, incorporándolas al discurso y prácticas biomédicas. Los niños terminan por ser objetualizados y despojados de la categoría de sujetos de derecho. Frente a este razonamiento, elaborado desde la lógica de la negatividad (HAN, 2014), vemos que la medicalización de la infancia produce cierto tipo de subjetividad. Sugerimos que dicha medicalización encamina a localizar la infancia en el arreglo normalidad-anormalidad y, en consonancia con ello, en el de salud mental-enfermedad mental, con el fin de que la subjetivación se realice desde el vértice infantil.

\section{I Los tres momentos de la medicalización infantil}

La medicalización de la infancia, fenómeno que adquirió rasgos específicos en la contemporaneidad, ha pasado por tres momentos, antes esbozados en el presente artículo.

En primera instancia, las psicociencias del siglo XIX, de la mano de la protopsiquiatría, vieron en la familia la explicación de la enfermedad mental. Aquí hallamos los inicios de la medicalización infantil. Este momento, que hemos denominado el germen de la medicalización infantil, se caracteriza por una mirada externalista; es el psiquiatra quien ausculta a la familia para identificar la trayectoria de la enfermedad de uno de sus miembros. La familia es, de esta forma, objetualizada.

El segundo momento, que llega con el psicoanálisis, retoma la explicación familiar de la patología psíquica, pero densificada con dos 
poderosas tecnologías conceptuales: el mundo interno y la historia personal. De la medicalización emerge un verse como niño traumatizado en donde podemos identificar un eje temporal conformado por dos polos: el primero, polo del adulto-niño, se refiere a la forma como los discursos psicoanalíticos y psicoterapéuticos conducen a que el sujeto adulto busque en su infancia los orígenes de su padecer. El segundo es el del niño-niño. Aun cuando Freud (1976) no trató directamente a niños, lo hizo de manera vicaria con el caso del pequeño Hans. Con Freud brotó una cascada de psicoanalistas $y$ psicoterapeutas expertos en infancia que constituyeron todo un campo especializado de intervención (MITCHELL; BLACK, 2004; BLEICHMAR, 1997; MITCHELL, 1993; GREENBERG; MITCHELL, 1983).

Este segundo momento del niño traumatizado se caracteriza por una mirada internalista totalizadora. El sujeto adul to o infantil es gobernado para que busque en su interior, su pasado y sus representaciones (conscientes o inconscientes) el origen de sus sufrimientos y la explicación de su presente. La totalidad de su historia es sometida a escrutinio.

El tercer momento, propio del presente, es el que define al sujeto infantil como niño autogestionado. La racionalidad que conduce la vida de los niños en la actualidad se funda en medicalizar todas las áreas y experiencias subjetivas; además de esto, tiende a evaluar todos los sectores de la vida del individuo como parte de su capital humano, el cual debe incrementarse ilimitadamente; $y$, finalmente, se cimenta en la norma según la cual cada ciudadano debe subjetivarse como empresa trabajando sobre sí mismo para conducirse como empresario de sí. La infancia es medicalizada en este sentido amplio por los psicocientíficos. De este modo, los administradores de la vida son guiados para detectar signos de inadecuación en los niños con el fin de introducir en el proceso formativo discursos de capital humano. Consecuentemente, la exigencia de capitalizar la propia vida desde la infancia misma aparece como un imperativo en la formación de los niños. La medicalización de la infancia produce una forma de subjetivación que llamamos niño autogestionado.

\subsection{De las causas de la medicalización de la infancia}

$\mathrm{Si}$ bien mostramos que el niño autogestionado es la figura de la subjetivación infantil en el presente, es preciso preguntarnos cómo hemos 
llegado a interpretar la infancia desde el horizonte de la anormalidad y, más aún, cómo llegamos a asociar anormalidad con patología. En este sentido, son tres las razones que explican la medicalización de la infancia: el arreglo veridiccional sobre la infancia realizado por las psicociencias, la incertidumbre en la administración de la vida infantil y la mercantilización de lo infantil.

Respecto del sistema veridiccional psicocientifico sobre la infancia, hallamos que estas disciplinas se han posicionado como tecnologías de gobierno (BEDOYA; CASTRILLÓN, 2018) que han permitido elaborar un sistema de verdades sobre lo humano para determinar qué es normal y qué no lo es y así mantener su prestigio en los círculos científicos, sociales y gubernamentales.

Las psicociencias han constituido un trípode justificador de la medicalización mediante la integración de la familia, el mundo interno y la historia personal como improntas de la subjetividad contemporánea. Deducimos que las psicociencias ubican a la infancia en el centro de la práctica medicalizadora apoyadas en la idea de que el sujeto psi, figura emblemática de la subjetividad que defienden, se constituye durante etapas tempranas de la historia personal. De este modo, convierten la infancia en la piedra de toque donde se estructuran los padecimientos y desviaciones. El cometido de las psicociencias es intervenir tanto para conducir la vida del infante y las relaciones que establece con su entorno (eje temporal niño-niño) como para reconducir al individuo adulto hacia su infancia (eje temporal adulto-niño) y encontrar allí la explicación de su malestar. La armazón veridiccional construida por Freud a partir de la idea de que el malestar del adulto es producto de vicisitudes traumáticas infantiles enfatiza en la historia primigenia del individuo y, por supuesto, conduce al sujeto a trabajar sobre ella para transformarse.

El descubrimiento de la infancia como campo de indagación psicológica tiene efectos de conducción de la acción de los individuos. A partir de esto siguieron una diversidad de discursos de verdad. Por tal razón, es factible proponer que en las ciencias psi confluyen aspectos políticos y científicos orientados al control de la población y que, como producto de ello, se construyen sistemas de clasificación en los que la infancia queda consolidada como objeto de vigilancia. Aunque corre 
la idea de que la categorización patológica de manuales como la CIE (Clasificación internacional de enfermedades) o el DSM (Manual diagnóstico $y$ estadístico de los trastornos mentales) dista del discurso psicoanalítico por su naturaleza categorial y taxativa (BIANCHI, 2014), otros autores sostienen que ambos defienden una misma posición: conformar cierto tipo de subjetividad, desde la pertenencia, a partir de técnicas de modificación del yo (BEDOYA, 2018; FRANCES, 2014).

Vemos al régimen de verdad creado por las psicociencias alimentarse del reconocimiento social y gubernamental que recibe, en su lógica de incluir dentro de sí sectores poblacionales cada vez más amplios. $\mathrm{Al}$ someter la infancia al proceso de psicologización, estos saberes esgrimen su poder; y al obtener reconocimiento, lo aumentan. La infancia es vista como la etapa crítica que determina la salud o la enfermedad futura. El ajuste y adaptación del sujeto infantil a las indicaciones del régimen de verdad sobre el desarrollo sano es la garantía de su salud mental.

Es justo decir que este sistema veridiccional psicocientifico sobre la infancia es el que fragua el segundo momento de la medicalización de la infancia, aquel en que la forma de subjetivación está definida por el verse como niño traumatizado. Al adoptar la tecnología conceptual del "mundo interno", las psicociencias acogieron la idea de que ese espacio profundo y caótico determina lo que somos. El trabajo sobre sí mismo para enfrentar los efectos traumáticos de la infancia resulta arduo, cuando no vano, y se presenta como toda una batalla del individuo consigo mismo que no puede evadirse so pena de destruir su propia vida. La terapia psi es el escenario y el producto que hay que consumir para sobrellevar tal experiencia agonística, a la que podemos definir como una experiencia de negatividad (HAN, 2014).

La incertidumbre en la administración de la vida infantil es la segunda razón que, a nuestro juicio, explica la medicalización de la infancia. Los administradores de la vida de los nińos han perdido los criterios para conducirlos (CHÁVEZ, 2014). En su práctica se instala una fuerte incertidumbre que los lleva a experimentar una sensación de soledad y culpa, caldo de cultivo para las mutuas increpaciones (MORALES et al., 2015; PEDRAZA; PERDOMO; HERNÁNDEZ, 2008; TIMIMI et al., 2004). En ese contexto, buscan amparo en el saber psi experto. 
Comprendemos que el vertiginoso aumento de consultas por parte de los padres a psicocientíficos, neurólogos y pedagogos se debe en parte a la búsqueda por resolver situaciones que no saben manejar en relación con los niños (VARGAS; PARALES, 2017; RABITO; CORREAS, 2014; BEDOYA; ALVIAR, 2013). Afrontan esta insuficiencia considerando al niño como anormal, cuando no un sujeto psicopatológico. La patologización representa un recurso importante para los administradores de la vida (VASEN, 2007).

El sujeto contemporáneo ha perdido los marcos ontológicos de referencia que le daban criterios para definir su acción (VÁZQUEZ, 2005; TAYLOR, 1996). La consecuencia es un repliegue sobre el propio yo auspiciado por los expertos psi, que con su lenguaje especializado indican al sujeto cuál es la vida apreciable como normal y cuál es la naturaleza de sus desviaciones, e informan a los administradores de la vida sobre cómo conducir su dirección de los otros y cómo guiar la conducta de sus dirigidos.

Respecto a la psicopatología infantil, el punto de inflexión se produjo con el DSM-III. Antes de esta versión, los trastornos mentales eran tratados como dificultades adaptativas manifiestas en las reacciones anormales a estímulos ambientales. De ahí que los problemas de la infancia relevantes para ese momento estuvieran vinculados a conductas socialmente reprobables: vandalismo, fuga o retraimiento (BIANCHI, 2014; FRANCES, 2014). Los problemas de aprendizaje aún no entraban en amplia consideración, aunque Lightner Witmer (1907) ya se había ocupado de estudiarlos a finales del siglo XIX.

El DSM-III abrió las puertas a la investigación neurobiológica. A partir de ese momento se asumió una trayectoria ajustada a los estándares de las disciplinas médicas, lo que tuvo efectos recíprocos sobre la psicopatología infantil. Al explicarlos desde etiologías neurobiológicas, los tratamientos psicoterapéuticos fueron desestimados al tiempo que se encumbraron las intervenciones psicofarmacológicas. En respuesta, nacieron múltiples movimientos antipsiquiátricos en Europa y Estados Unidos (BENTALL, 2014).

Debe destacarse el ascenso de Reagan a la presidencia durante esos mismos años como hecho fundamental en la historia de la salud mental debido a su propuesta de disminuir al máximo posible la injerencia federal 
en los asuntos públicos, lo que dejó a la deriva un sinnúmero de instituciones antes mantenidas por políticas de bienestar (THOMAS, 1998). Tal abandono llevó a la privatización y a concebir la vida humana como un negocio.

De la neuropsiquiatrización al manejo mediante psicofármacos hubo un solo paso. La asistencia diagnóstica médica-psicocientífica adquiere aquí su sentido (TIMIMI, 2010). Pero mientras el diagnóstico reduce la incertidumbre en padres y maestros (MORALES et al., 2015; BRZOZOWSKI, 2009), la culpa que experimentan no cesa, pues les quedan irresueltas sus dudas respecto a la responsabilidad de los administradores infantiles en la condición médica del niño. Los guías infantiles terminan así convertidos en pacientes del dispositivo normativo e interventivo dispuesto por los científicos psi.

En esta segunda explicación de la medicalización de la infancia se conserva la figura del niño traumatizado. Con el diagnóstico, la familia enfrenta su propia disfuncionalidad y queda enganchada en las lógicas del gobierno psicocientífico.

Asu vez, permanece la lógica de la negatividad porque los administradores entran en un triple combate: consigo mismos (debido a la resistencia que les genera el responsabilizarse por la condición del niño), con el infante (quien, a pesar del diagnóstico, aún reta sus formas de entendimiento y acción cotidianas) y con los expertos (quienes imponen unas demandas técnicas difíciles de satisfacer).

Los niños viven su propio combate con los administradores de su vida, quienes se esfuerzan por conducirlos bajo la luz de la normalidad para evadir cualquier desviación posible. De esta manera, el repliegue sobre el propio yo, consecuencia de la pérdida de los marcos ontológicos de referencia, es un centramiento agonístico en el yo tanto de los nińos como de los encargados de gobernarlos.

La tercera causa de la medicalización contemporánea de la infancia es la mercantilización de lo infantil. El neoliberalismo, experto en convertir cada espacio subjetivo y social en mercadeable, hizo de la infancia y la psicopatología poderosos nichos de mercado gracias a la estrategia de medicalización que las psicociencias inventaron. Visto desde otra perspectiva, mientras lo psi es creado por el liberalismo, el neoliberalismo lo mercantiliza. 
La infancia, incluida mediante su medicalización en la práctica de gobierno biopolítico liberal (FOUCAULT, 2000) bajo el signo de la negatividad (HAN, 2014), es medicalizada positivamente en el neoliberalismo para asegurar una subjetividad emprendedora. Queda mercantilizada tanto la enfermedad como la salud: la primera, debido a lo que hemos denominado gestión positiva de la enfermedad; la segunda, a través de la gestión preventiva de la salud. En ambos casos, que haya gestión implica una mercantilización de la infancia, sea que el nińo reciba un diagnóstico o no. Engancharse en el mercado psicocientífico (ILLOUZ, 2007, 2010) y biomédico del mejoramiento ilimitado de sí (ROSE, 2012) es el corazón mismo de la gestión positiva de la salud. Como lo dice Barcala (2015, p. 79):

Las políticas de salud, enmarcadas dentro de una poderosa ideología neoliberal constructora de subjetividades, colaboraron en la deconstrucción de la condición de ciudadano como sujeto de derecho para convertirla en otra, la de consumidor/cliente, y el acceso a los cuidados de la salud pasó de ser un derecho adquirido a ser una mercancía, posible de comprar según el poder adquisitivo de cada hogar.

Lo farmacológico ofrece una solución rápida y eficaz a una sociedad atravesada por el discurso del empresario de si (ARIZAGA; FARAONE, 2008). Esto explica que los gobernantes no le teman a la enfermedad mental. Mientras que la protopsiquiatría razonaba lógicas de exclusión apoyada en el modelo de encierro disciplinario (FOUCAULT, 1967, 2005) - en tanto que la locura era una alteridad temida -, la medicalización contemporánea acoge al enfermo mental para aprehenderlo y normalizarlo. El neoliberalismo no le teme al enfermo mental porque es posible hacer una gestión positiva mercantilizando su padecer. Bajo la misma estrategia, los temores que produce la infancia son manejados con la medicalización de la experiencia de los niños, patologizándola y encumbrándola como un poderoso nicho de mercado.

Para algunos críticos, esta nueva forma de disciplinar a los niños y de ejercer control poblacional demanda poco gasto de personal y les genera ganancias cuantiosas a las empresas farmacéuticas. El asunto gana peso al quedar manifiesto que esas mismas entidades financian y participan en los estudios que avalan los manuales diagnósticos (FARAONE et al., 2009).

Si bien numerosos estudios denuncian que la medicalización de la infancia está fuertemente dirigida por los intereses comerciales de las 
farmacéuticas (DUEÑAS, 2019; BARCALA, 2015; STOLKINER, 2012; BARROS, 2008), nosotros sugerimos destacar otros actores que acumulan prestigio, ganancias y públicos vinculados a ellos cuando se medicaliza la vivencia de los niños, como el gremio de psicocientíficos. No solo obtienen un progresivo reconocimiento social y gubernamental, sino que también acumulan un gran poder al monopolizar el diagnóstico psicológico. Además, los científicos psi son generosos al donar a la sociedad su sistema de verdades para hacer más gobernables a los menores (ROSE, 1996, 2012).

Un elemento poco mencionado en aquellos estudios es el uso estratégico de la medicalización infantil para efectos del control poblacional en términos de vigilancia, o, con más precisión, el control sin vigilancia del que tanto Foucault (1985) como Deleuze (1999) fueron conscientes. Para estos autores, ya no habitamos sociedades disciplinarias, sino sociedades de control cuya meta es conducir la población sin que se sienta vigilada: autovigilancia, según el diagnóstico de Han $(2013,2014)$. En la medida en que se naturaliza la medicalización de la infancia, la experiencia de los niños y de sus cuidadores se visibiliza. Al hablar abiertamente de sus condiciones, los individuos devengan transparentes a sí mismos y a los otros. Los niños van siendo guiados por sus administradores para elegir controlarse mediante la autovigilancia. La transparencia es la condición de posibilidad del empresarismo de sí, bajo el cual está formado el sujeto desde su niñez.

Lo anterior nos permite concluir dos cosas: por una parte, el ideal de subjetividad infantil que el neoliberalismo despliega a partir de la medicalización de la infancia es el del niño formado como emprendedor y abierto a la gestión positiva (mercantilizada) de su vida con la que los administradores de la vida infantil quieren seducirlo. A esta forma de subjetividad la hemos denominado el niño autogestionado. Por otra parte, advertimos que en ella se manifiesta una lógica de la positividad, pues los administradores de la vida de los niños se alienan voluntariamente al mercado que produce la medicalización de la infancia y luchan para que los niños hagan lo propio.

\section{Consideraciones finales}

Hemos presentado los resultados de un estudio teórico. En él nos preguntamos por la manera como las psicociencias, en la era del capitalismo 
neoliberalizado, producen unas formas de subjetividad. Aquí queremos lanzar una tesis: las prácticas de gobierno, en cualquier época, se han ocupado de la eliminación de la alteridad, de lo extraño. Particularmente, la racionalidad de gobierno neoliberal le teme a la alteridad, pero no a la anormalidad.

Sostenemos, con Vázquez (2005) y Taylor (1996), que un rasgo central de nuestra época es la pérdida de los marcos ontológicos de referencia (MOR), lo que ha producido un repliegue del individuo sobre su yo y su dependencia respecto de la experticia psicocientífica, biomédica y psicofarmacológica. Postulamos una segunda consecuencia: con la pérdida de los MOR, tanto el individuo como quienes lo gobiernan se ven enfrentados con la alteridad, con lo-otro inaprehensible, y buscan reconducirlo hacia la normalidad. La normalización de lo anormal ha marcado nuestra forma de relacionarnos con el mundo (LE BLANC, 2004, 2010).

En este punto se inserta la estrategia de gobierno medicalizadora y la labor de gobierno que realizan los administradores de la vida de los niños.

La enorme dificultad que representa lo infantil para su comprensión y su manejo - lo cual indica su carácter de alteridad respecto del mundo de los adultos - es confrontada mediante un ejercicio normalizador, es decir, de reconducción de lo-extraño hacia lo-familiar. Cuando no es posible, y eso otro que es la alteridad del niño se resiste a ser aprehendida, el administrador patologiza lo inaprehensible. Toda una psiquiatrización de la alteridad toma lugar. Pero, como indica Le Blanc (2004), no se soluciona nada, pues lo patológico aparece simplemente como variación de lo-mismo (lo normativo) y no como verdadera manifestación de la alteridad. Dentro de este contexto, la labor de gobierno de la vida de los niños que realizan los administradores de la vida, los psicocientíficos, los Estados y los medios de comunicación consiste en patologizar la alteridad infantil y, de ese modo, normalizarla.

Una vez medicalizada la vida de los niños, queda expuesta al mercado del mejoramiento ilimitado. Se les proporciona todo tipo de productos a los nińos diagnosticados con algún trastorno, a aquellos que no tienen diagnóstico psicopatológico y a sus administradores, para perpetuar la producción de empresarios de sí mismo. El neoliberalismo se apropia de la anormalidad infantil devenida enfermedad al crear todo un mercado a su alrededor. Sin embargo, también le teme porque ella quiebra tanto la lógica de quienes pretenden gobernar a los niños como la lógica misma del neoliberalismo. 
Normalizar la vida y crear un poderoso mercado de lo infantil (incluida la patología de los niños) son los dividendos que el neoliberalismo obtiene. Pero, para alcanzar su cometido, el neoliberalismo requiere del compromiso de amplios sectores de las psicociencias contemporáneas.

\section{Referencias}

ÁLVAREZ-URÍA, F. Viaje al interior del yo. La psicologización del yo en la sociedad de los individuos. In: CASTEL, R.; RENDUELES, G.; DANZELOT, J.; ÁlVAREZ-URÍA, F. (org.). Pensar y resistir. La sociología crítica después de Foucault. Madrid: Ediciones Ciencias Sociales; Círculo de Bellas Artes, p. 101-134, 2006.

AMORÓS, V. Psicología y psicoterapia humanística. Revista de Psicología de la PUCP, Lima, v. 14, n. 1, p. 73-80, oct. 1996.

ARIZAGA, M.; FARAONE, S. La medicalización de la infancia: niños, escuela y psicotrópicos. Informe final. Observatorio Argentino de Drogas, Sedronar - Instituto de Investigaciones Gino Germani. Buenos Aires: Universidad de Buenos Aires, 2008.

BARCALA, A. La medicalización de la niñez: prácticas en Salud Mental y subjetividad de niñas, niños y adolescentes con sufrimiento psicosocial. In: BARCALA, A.; LUCIANI CONDE, L. (org.). Salud Mental y niñez en la Argentina. Legislaciones, políticas y prácticas. Buenos Aires: Teseo, p.75-105, 2015.

BARROS, J. Nuevas tendencias de la medicalización. Ciência \& Saúde Coletiva, v. 13, p. 579$587,2008$.

BEDOYA, M. La gestión de sí mismo. Ética y subjetivación en el neoliberalismo. Medellín: Editorial Universidad de Antioquia, 2018.

BEDOYA, L.; ALVIAR, M. Familias entrampadas en la hiperactividad. Revista Facultad de Trabajo Social, v. 29, n. 29, p. 237-259, 2013.

BEDOYA, M.; CASTRILLÓN, A. Psicociencias y gobierno de la subjetividad. Iatreia, v. 31, n. 1, p. 18-28, 2018.

BENTALL, R. Medicalizar la mente. ¿¿Sirven de algo los tratamientos psiquiátricos? Barcelona: Herder, 2014.

BIANCHI, E. Todo tiene un principio... y en el principio fue el DSM-III. El desbloqueo epistemológico y tecnológico de la psiquiatría biológica estadounidense. Revista Culturas Psi/Psy Cultures, v. 2, p. 87-114, 2014.

BLEICHMAR, N.; DE BLEICHMAR, C. El Psicoanálisis después de Freud. Ciudad de México: Paidós, 2017.

BROWN, W. El pueblo sin atributos. Barcelona: Malpaso, 2017. 
BRZOZOWSKI, F. S. Transtorno de déficit de atenção com hiperatividade: medicalização, classificação e controle dos desvios. 2009. 90 f. Disertación (Maestría en Salud Pública) - Programa de Pós-Graduação em Saúde Pública, Universidad Federal de Santa Catarina, Florianópolis, 2009.

CABANAS, E.; ILLOUZ, E. Reseña HAPPYCRACIA. Cómo la ciencia y la industria de la felicidad controlan nuestras vidas. Barcelona: Paidós, 2019.

CABRAL DE BARROS, J. Ampliando espaços para a "medicalização": instrumento para desfrute de melhores níveis de saúde? In: CAPONI, S.; VERDI, M.; BRZOZOWSKI, F. S.; HELLMANN, F. (org.). Medicalizaçáo da Vida: Ética, Saúde Pública e Indústria Farmacêutica. Palhoça: Unisul, p. 89-95, 2010.

CANGUilheM, G. Le cerveau et la pensée. Prospective et Santé, v. 14, p. 81-98, 1980.

CANGUILHEM, G. ¿Qué es la psicología? Revista colombiana de psicología, v. 7, p. 7-14, 1998.

CAPONI, S. Michel Foucault e a persistência do poder psiquiátrico. Ciência \& Saúde Coletiva, v. 14, n. 1, p. 95-103, 2009a.

CAPONI, S. Para una genealogía de la anormalidad: la teoría de la degeneración de Morel. Scientiæ studia, v. 7, n. 3, p. 425-45, 2009b.

CAPONI, S. Un análisis epistemológico del diagnóstico de depresión. Comunicação saúde educaçáo, v. 13, n. 29, p. 327-38, 2009c.

CAPONI, S. Emil Kraepelin y el problema de la degeneración. História, Ciências, Saúde, v. 17, n. 2, p. 475-494, 2010a.

CAPONI, S. Georges Canguilhem: del cuerpo subjetivo a la localización cerebral. Salud Colectiva, v. 6, n. 2, p- 149-161, 2010b.

CAPONI, S. Clasificaciones, acuerdos y negociaciones: bases de la primera estadística internacional de enfermedades mentales (París, 1889). Dynamis, v. 32, n. 1, p. 185-207, 2012 a.

CAPONI, S. Loucos e degenerados: uma genealogia da psiquiatria ampliada. Río de Janeiro: Fiocruz, 2012b.

CASTEL, F.; CASTEL, R.; LOVELL, A. (org.). La sociedad psiquiátrica avanzada. El modelo norteamericano. Barcelona: Anagrama, 1980.

CASTEL, R. Homo psicologicus. Entrevista a Robert Castel por Daniel Friedmann. Revista de la Asociación Espańola de Neuropsiquiatría, v. 6, n. 18, p. 454-462, 1986.

CASTEL, R. El orden psiquiátrico. La edad de oro del alienismo. Buenos Aires: Nueva Visión, 2009.

CASTRO, E. Diccionario Foucault. Temas, conceptos y autores. Buenos Aires: Siglo XXI, 2011.

CASTRO-GÓMEZ, S. Historia de la gubernamentalidad II. Filosofía, cristianismo y sexualidad en Michel Foucault. Bogotá: Siglo del Hombre, 2016.

CASTRO ORELLANA, R. Psicologización de la vida. Lectura del Curso de Foucault Le Pouvoir Psychiatrique. Logos. Anales del seminario de Metafísica, v. 47, p. 55-79, 2014. 
CHÁVEZ, T. Análisis del fenómeno del TDAH (Trastorno por déficit de atención con hiperactividad) en la Dirección de Psicopedagogía de la Secretaría de Educación Jalisco. Los intentos de hacer invisible la subjetividad en los ambientes escolares. Diálogos sobre Educación, v. 8 , n. 5 , p. 1-15, 2014.

DELEUZE, G. Conversaciones 1972-1990. Valencia: Pre-textos, 1999.

DELEUZE, G. La subjetivación: curso sobre Foucault III. Buenos Aires: Cactus, 2015.

DREYFUS, H.; RABINOW, P. Michel Foucault. Más allá del estructuralismo y la hermenéutica. Buenos Aires: Nueva Visión, 2001.

DUEÑAS, G. Acerca de la patologización y medicalización de los malestares infantiles actuales y sus derechos en juego. Derechos en Acción, v. 12, n. 12, p. 304, 2019.

FARAONE, S.; BARCALA, A.; BIANCHI, E.; TORRICELLI, F. La industria farmacéutica en los procesos de medicalización/medicamentalización en la infancia. Margen, v. 54, p. 1-10, 2009.

FERNÁNDEZ, M. La ideología de la neurociencia. Temas de psicoanálisis, v. 8, p. 1-27, 2014.

FORCADES, T. Los crímenes de las grandes compañías farmacéuticas. Cuadernos CJ, v. 141, p. $1-42,2006$.

FOUCAULT M. Historia de la locura en la época clásica. México: Fondo de Cultura Económica, 1967.

FOUCAULT, M. Saber y verdad. Madrid: La Piqueta, 1985.

FOUCAULT, M. ¿Qué es la Ilustración? [Qu’est-ce que les Lumières?]. Actual, n. 28, p.1-18, 1994

FOUCAULT, M. La vida de los hombres infames. La Plata: Altamira, 1996.

FOUCAULT, M. Historia de la sexualidad. Vol. II. El uso de los placeres. Madrid: Siglo XXI, 1998.

FOUCAULT, M. Estética, ética y hermenéutica. Barcelona: Paidós, 1999.

FOUCAULT, M. Los anormales. Curso en el Collège de France (1974-1975). Buenos Aires: Fondo de Cultura Económica, 2000.

FOUCAULT, M. El sujeto y el poder. In: DREYFUS, H.; RABINOW, P. (org.). Michel Foucault. Más allá del estructuralismo y la hermenéutica. Buenos Aires: Nueva Visión, p. 241-260, 2001.

FOUCAULT, M. La hermenéutica del sujeto. Curso en el Collège de France (1981-1982). México: Fondo de Cultura Económica, 2002.

FOUCAULT, M. El poder psiquiátrico. Curso en el Collège de France (1973-1974). Buenos Aires: Fondo de Cultura Económica, 2005.

FOUCAULT, M. Nacimiento de la biopolítica. Curso en el Collège de France (1978-1979). Buenos Aires: Fondo de Cultura Económica,2007. 
FOUCAULT, M. El gobierno de sí y de los otros. Curso en el Collège de France (1982-1983). Buenos Aires: Fondo de Cultura Económica, 2009.

FRANCES, A. ¿Somos todos enfermos mentales? Manifiesto contra los abusos de la Psiquiatría. Madrid: Ariel, 2014.

FREUD, S. Análisis de la fobia de un niño de 5 años. In: ETCHEVERRY, J. L. Obras completas: Sigmund Freud. Vol 10. Buenos Aires: Amorrortu, p.1-118, 1976.

GARCÍA, M. Espacio y Poder. El espacio en la reflexión de Michel Foucault. México: Universidad Autónoma Metropolitana, 2006.

GOTZSCHE, P. Medicamentos que matan y crimen organizado. Barcelona: Los libros del lince, 2014.

GREenberG, J.; MITCHELL, S. Object Relations in Psychoanalytic Theory. Cambridge: Harvard University Press, 1983.

HAN, B-C. La sociedad de la transparencia. Barcelona: Herder, 2013.

HAN, B-C. Psicopolítica. Neoliberalismo y nuevas técnicas de poder. Barcelona: Herder, 2014.

ILLOUZ, E. Intimidades congeladas. Las emociones en el capitalismo. Madrid: Katz, 2007.

ILLOUZ, E. La salvación del alma moderna. Terapia, emociones y cultura de la autoayuda. Madrid: Katz, 2010.

KANT, I. ¿Qué es la Ilustración? Foro de Educación, n. 11, p. 249-254, 2009.

LAVAL, C.; DARDOT, P. La nueva razón del mundo. Ensayo sobre la sociedad neoliberal. Barcelona: Gedisa, 2013.

LAVAL, C.; DARDOT, P. Común. Ensayo sobre la revolución en el siglo XXI. Barcelona: Gedisa, 2015 .

LAVAL, C.; DARDOT, P. La pesadilla que no acaba nunca: el neoliberalismo contra la democracia. Barcelona: Gedisa, 2017.

LE BLANC, G. Canguilhem y las normas. Buenos Aires: Nueva Visión, 2004.

LE BLANC, G. Las enfermedades del hombre normal. Buenos Aires: Nueva Visión, 2010.

MARTÍNEZ, A. A medicalizaçáo dos estados de ânimo. O consumo de antidepressivos e as novas biopolíticas das afliçóes. In: CAPONI, S.; VERDI, M.; BRZOZOWSKI, F. S.; HELLMANN, F. (org.). Medicalizaçáo da Vida: Ética, Saúde Pública e Indústria Farmacêutica. Palhoça: Unisul, p.111-134, 2010.

MASLOW, A. El hombre autorrealizado. Barcelona: Paidós, 1989.

MITCHELL, S. A. Conceptos relacionales en psicoanálisis: Una integración. México: Siglo XXI, 1993.

MITCHELL, S. A.; BLACK, M. Más allá de Freud: una historia del pensamiento psicoanalítico moderno. España: Herder, 2004. 
MORALES, C.; GARCÍA, M.; ÁlVAREZ, C.; GERVÁS, M.; PARDO, R.; PÉREZ, O.; DE LA TORRE, M.; SANTACREU, M. Guía de intervención clínica infantil guía desarrollada por el Centro de Psicología Aplicada (CPA). 2015. Disertación. Universidad Autónoma de Madrid, Madrid, 2015.

PAPALINI, V. “Tecnologías del yo”: entre la gubernamentalidad y la autonomía. In: RODRÍGUEZ FREIRE, R. (org.). El gobierno del presente. Materiales críticos. Valparaíso: Instituto de Literatura y Ciencias del Lenguaje. Pontificia Universidad Católica de Valparaíso, p.253-275, 2013.

PEDRAZA, S.; PERDOMO, M.; HERNÁNDEZ, N. (org.). Terapia narrativa en la coconstrucción de la experiencia y el afrontamiento familiar en torno a la impresión diagnóstica de TDAH. Universitas Psychologica, v. 8, n. 1, p. 199-214, 2008.

PIGNARRE, P. El gran secreto de la industria farmacéutica. Barcelona: Gedisa, 2005.

RABITO, M. F.; CORREAS, J. Guías para el tratamiento del Trastorno por Déficit de Atención e Hiperactividad: una revisión crítica. Actas espańolas de psiquiatría, v. 42, n. 6, p. 315-324, 2014.

ROSE, N. Inventing our selves. Psychology, Power and Personhood. Cambridge: Cambridge University Press, 1996.

ROSE, N. Normality and pathology in a biological age. Outlines. Critical Practice Studies, v. 3, n. 1, p. 19-33, 2001.

ROSE, N. Psicologia como uma ciência social. Psicologia \& Sociedade, v. 20, n. 2, p. 155-164, 2008.

ROSE, N. Políticas de la vida. Biomedicina, poder y subjetividad en el siglo XXI. La Plata: Unipe, 2012 .

ROSE, N.; NOVAS, C. Biological citizenship. In: ONG, A.; COLLIER, S. (org.). Blackwell companion to global anthropology. Oxford: Blackwell, p.439-463, 2004.

SENNETT, R. La corrosión del carácter. Las consecuencias personales del trabajo en el nuevo capitalismo. Barcelona: Anagrama, 2000.

STOLF, F; CAPONI, S. Medicalização dos desvios de comportamento na infância: aspectos positivos e negativos. Psicologia: Ciência e profissáo, v. 33, n. 1, p. 208-221, 2013.

STOLKINER, A. Infancia y medicalización en la era de "la salud perfecta". Propuesta Educativa, v. 1, n. 37 , p. $28-38,2012$.

SY, A. La medicalización de la vida: hibridaciones ante la dicotomía Naturaleza/Cultura. Ciência \& Saúde Coletiva, v. 23, p. 1531-1539, 2018.

TAYLOR, C. Las fuentes del yo. La construcción de la identidad moderna. Barcelona: Paidós, 1996.

THOMAS, A. Ronald Reagan and the commitment of the mentally ill: Capital, interest groups, and the eclipse of social policy. Electronic Journal of Sociology, v. 3, n. 4, 1998. 
La medicalización de la infancia como estrategia de gobierno | Mauricio Bedoya-Hernández, Pablo Andrés de Francisco-León, Lina Julietd Mesa-Osorio

TIMIMI, S. The McDonaldization of childhood: Children's mental health in neo-liberal market cultures. Transcultural Psychiatry, v. 47. n. 5, p. 686-706, 2010.

TIMIMI, S.; MONCRIEFF, J.; JUREIDINI, J.; LEO, J.; COHEN, D.; WHITFIELD, C. A critique of the International Consensus Statement on ADHD. Clinical Child and Family Psychology Review, v. 7, n. 1, p. 59-63, 2004.

URABAYEN, J.; CASERO, J. La gubernamentalidad biopolítica: de la sociedad de control estatal al liberalismo. Co-herencia, v. 15, n. 29, p. 67-92, 2018.

VARGAS, A.; PARALES, C. La Construcción Social de la Hiperactividad. Revista Colombiana de Psicología, v. 26, n. 2, p. 245-262, 2017.

VÁZQUEZ, F. "Empresarios de nosotros mismos". Biopolítica, mercado y soberanía en la gubernamentalidad neoliberal. In: UGARTE, J. (org.). La administración de la vida. Estudios biopolíticos. Barcelona: Anthropos, p. 73-103, 2005

VASEN, J. C. La atención que no se presta: el "mal" llamado ADD. Buenos Aires: Novedades Educativas, 2007.

WITMER, L. Clinical Psychology. Psychological Clinic, v. 1, p. 1-9, 1907.

Recebido: 30/06/2020

Aceito: 02/08/2020 


\section{The medicalization of childhood as a government strategy}

\section{Abstract}

This paper provides an account of a study whose aim was to know the psychoscientific strategies associated with the production of subjectivity in neoliberalism. The authors used a historicalcritical approach based on the foucauldian studies. Results: ( 1 ) the medicalization of life has been a government strategy; (2) the medicalization of childhood -also a government strategy- has gone through three stages, each of which has produced a specific form of subjectivity; (3) three reason for the medicalization of childhood have been found, and (4), psychoscience and bioscience are the discourses that have directed this medicalization. The authors conclude that neoliberalism has used this medicalization strategy, which was created by the psy sciences, to expand the commodification of life and configure the self-managed subjectivity of the entrepreneur.

Keywords: Medicalization. Childhood. Subjectivity. Neoliberalism.

\section{A medicalização da infância como estratégia de governo}

\section{Resumo}

Este artigo informa sobre a pesquisa cujo intuito foi conhecer as estratégias psicocientíficas associadas com a produção de subjetividade no neoliberalismo. O método empregado foi o histórico-crítico fundamentado nos estudos foucaultianos. Resultados: ( I ) a medicalização da vida tem sido uma estratégia de governo; (2) a medicalização da infância - também estratégia de governo - tem passado por três momentos, cada um deles produz uma forma específica de subjetividade; (3) foram descobertas três razões para medicalizar a infância e (4) as psicociências e as biociências são os discursos que têm orientado tal medicalização. Conclui-se que o neoliberalismo tem empregado esta estratégia de medicalização elaborada pelas ciências psi para ampliar a mercantilização da vida e configurar a subjetividade autogerenciada dos empreendedores.

Palavras-chave: Medicalização. Infância. Subjetividade. Neoliberalismo. 